\title{
Optical Absorption and Scattering Properties of The Active Layer Of Perovskite Solar Cells Incorporated Silver Nanoparticles
}

\author{
PANUWAT CHAIYACHATE ${ }^{1}$ and THANANCHAI DASR ${ }^{2,3^{*}}$ \\ 'School of Science and Mathematics, Faculty of Industrial and Technology, Rajamangala \\ University of Technology Isan Sakon Nakhon Campus, Sakon Nakhon, 47160, Thailand. \\ ${ }^{2}$ Faculty of Applied Science and Engineering, Khon KaenUniversity, Khon Kaen, 40002, Thailand. \\ ${ }^{3}$ The Natural Resources, Energy and Environmental Research Sector (NEE), \\ Khon Kaen University, Khon Kaen, 40002, Thailand. \\ "Corresponding author E-mail: thananchai_dasri@ hotmail.com \\ http://dx.doi.org/10.13005/ojc/330228
}

(Received: February 11, 2017; Accepted: April 04, 2017)

\begin{abstract}
This article presents the optical properties of pure silver $(\mathrm{Ag})$ nanoparticle embedded in perovskite layer of perovskite solar cells using the discrete dipole approximation method (DDA). The influences of the Ag nanosphere size, shapes, and direction of the polarization direction of incident light respect to the particle axis on the absorption and scattering efficiencies on the localized surface plasmon resonance (LSPR) were investigated. For the single Ag nanoparticle, the calculated absorption and scattering spectra with increasing Ag diameter from 10 to $40 \mathrm{~nm}$, both absorption and scattering resonance peaks were found at the position of around $624 \mathrm{~nm}$. However, the optical efficiencies increases further with increasing the particle size up to $40 \mathrm{~nm}$. For the optical properties of the two Ag nanoparticles with fixed $20 \mathrm{~nm}$ diameter and varying inter-particles spacing, the aligning particles perpendicular to both the propagation and polarization direction of light the increasing of the inter-particles spacing produces the shorter wavelength shift of the position peaks. In contrast, aligning particles perpendicular to the propagation direction and parallel to the polarization direction of light they were slightly shifted to longer wavelength as the inter-particles spacing increase. Finally for single Ag nanowire with varying the length of nanowire from 36.88 to $104.40 \mathrm{~nm}$, both the absorption and scattering efficiencies peaks position get longer wavelength linearly shifted when the particlesax is was aligned perpendicular to the propagation direction and parallel to the polarization direction of light. However, they shift to shorter wavelength when the particlesax is was aligned both perpendicular to the propagation direction and the linear polarized light.
\end{abstract}

Keywords: Plasmonic nanoparticles, Perovskite solar cell, Discrete dipole approximation 


\section{INTRODUCTION}

The metal nanoparticles (NPs) such as gold $(\mathrm{Au})$, silver $(\mathrm{Ag})$ and copper $(\mathrm{Cu})$ which possess a negative real and small positive imaginary dielectric constant in thin Ims or nanostructures are potential candidates in many applications such as sensors ${ }^{1}$, organic light-emitting devices (OLEDs) ${ }^{2}$ and organic solar cells ${ }^{3-6}$ due to their localized surface plasmon resonance(LSPR). LSPRs are collective oscillations of a conductor's surface electrons at the interface between the metal NPs which much smaller than incident light wavelength and a dielectric medium under excited electromagnetic field that tend to trap optical waves near their interface ${ }^{7}$. The operation of the plasmonic nanoparticles in the above applications is affected by the refractive index of the metal and the surrounding medium, the particle size and shape as well as the polarization direction of the incident light. In the case of the plasmonic nanoparticles shape, variety of shape such as nanospheres ${ }^{3,8-11}$, nanocubes ${ }^{11}$, nanoprisms ${ }^{12}$, nanoflowers ${ }^{3,13}$ and nanorod $^{3,11,14}$ have been reported as the source for generating LSPRs. Perovskite solar cells (PSCs), known as photovoltaic device, have been reported that they have rapidly attracted attention due to its advantageous features including less expensive material for fabricating PSCs, and rapidly increasing device efficiency ${ }^{15-19}$. Recently, plasmonic nanoparticles have been reported that utilizing them in PSCs can improvethe light absorptionin perovskite material ${ }^{18-22}$. Various types of metal NPs like pure nanospheres ${ }^{21}$, core/shell nanospheres and nanorods ${ }^{18,19}$ were reported. This article will theoretically present the effect of LSPR introduced by Ag NPs on the optical properties in the active layer of PSCs by using discrete Dipole Approximation (DDA). It is one of the most frequently used numerical methods for computing optical properties such as scattering and absorption by arbitrary size, shape nano-geometry ${ }^{22}$. The study will be presented the influence of the geometry and concentration of Ag NPs on the optical properties. This study will contribute to better understanding the uses of metal NPS for enhancing PSCs performances.

\section{EXPERIMENTAL}

\section{Computational details}

The absorption and scattering efficiencies of the pure Ag NPs were simulated based on the DDA theory ${ }^{22}$. This theory, the object in arbitrary shape is replaced with an assembly of $N$ point dipoles in which the polarizability and positions are specified as $\alpha_{i}$ and
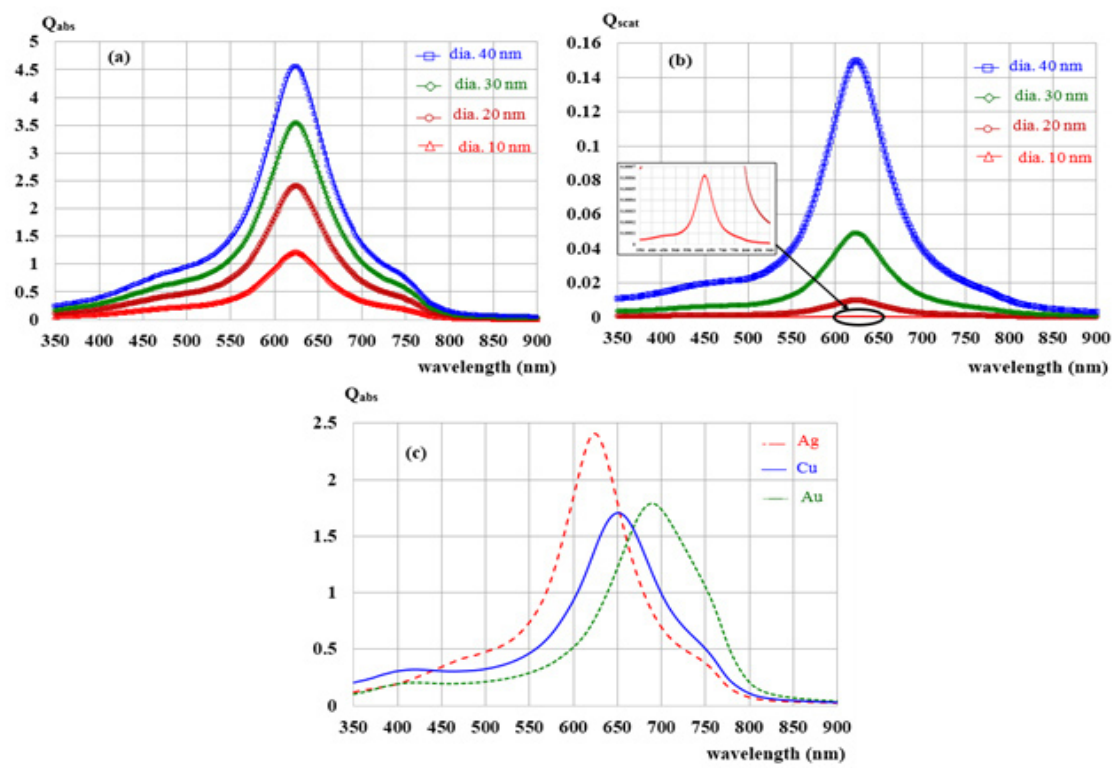

Fig. 1: (a) The absorption efficiency and (b) scattering efficiency for the Ag nanoparticle with varying their sizes. (c) Absorption efficiency for $\mathrm{Ag}$, $\mathrm{Au}$ and $\mathrm{Cu}$ nanoparticles of a $10 \mathrm{~nm}$ radius. The insets in (a) shows the spectra in selected wavelength length. 
$r_{i}$, respectively. The polarization induced $\mathbf{P}_{\mathbf{i}}$ in each particle in the presence of an applied field is then described by Eq.(1):

$$
\overrightarrow{\mathrm{P}}_{\mathrm{i}}=\alpha_{i} \overrightarrow{\mathrm{E}}_{\mathrm{loc}}\left(\overrightarrow{\mathrm{r}}_{i}\right)
$$

where $_{\text {loc }}$ is the sum of the incident field and the contribution from all other $\mathrm{N}-1$ dipoles. When this polarization is obtained, the absorption, scattering and extinction cross sections of light can be calculated by Eqs. (2)- (4):

$$
\begin{aligned}
& C_{e x t}=\frac{4 \pi k}{\left|E_{0}^{2}\right|} \sum_{i=1}^{N} \operatorname{Im}\left(\overline{\mathrm{E}}_{i x c, i}^{*} \cdot \overline{\mathrm{P}}_{\mathrm{i}}\right), \\
& C_{a b s}=\frac{4 \pi k}{\left|E_{0}^{2}\right|} \sum_{i=1}^{N}\left\{\operatorname{Im}\left[\overline{\mathrm{P}}_{\mathrm{i}} \cdot\left(\alpha_{i}^{-1}\right)^{*} \overline{\mathrm{P}}_{i}^{*}\right]-\frac{2}{3} k^{3}\left|\overline{\mathrm{P}}_{i}\right|^{2}\right\}, \\
& C_{s c a t}=C_{e x t}-C_{a b s} .
\end{aligned}
$$

Equations (2) and (3), “*' symbol stands for the conjugate of a complex variable, kis the wave number and $E_{0}$ is the amplitude of incident electric field. And scattering, absorption and extinction efficiencies can be calculated by $Q_{s c a t}=C_{s c a} / \pi R^{2}$, $Q_{e x t}=C_{e x t} / \pi R^{2}$ and $Q_{a b s}=C_{a b s} / \pi R^{2}$, respectively. $R$ is radius of spherical nanoparticle. The dimensions of the morphologies Ag NPs were taken from Pathaket al. ${ }^{18}$. The refractive indices of Methylammonium lead triiodide $\left(\mathrm{CH}_{3} \mathrm{NH}_{3} \mathrm{Pbl}_{3}\right)$ were taken from Löperet al. ${ }^{23}$. The dielectric constants for metal NPS were obtained by using Lorentz-Drude model ${ }^{24}$.

\section{RESULTS AND DISCUSSIONS}

The following results are the absorption and scattering efficiencies of Ag nanoparticles consisting of a single homogeneous spherical particle, two homogeneous sphericalparticles and nano wire at a fixed the surrounding medium refractive indices of $\mathrm{CH}_{3} \mathrm{NH}_{3} \mathrm{Pbl}_{3}$. At the first stage of this research,
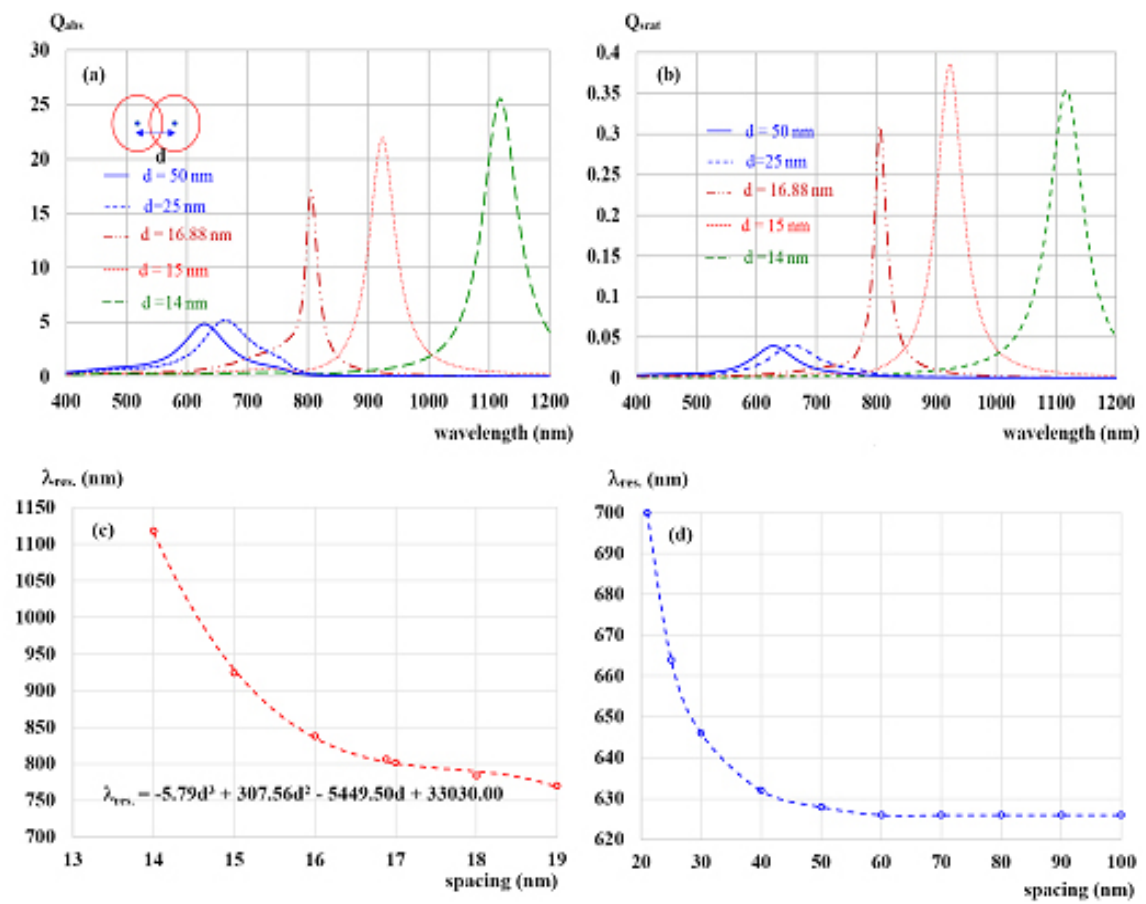

Fig. 2: Calculation of the spectral: (a) absorption and (b) scattering efficiencies as function of wavelength for two closely spaced Ag spherical particles, aligned perpendicular to the propagation direction and parallel to the linear polarized light. The spacing between two particles are indicated in the inset. (c) Shows effect of the overlapped and (d) non- overlapped distance between two Ag nanoparticles and the absorption peak position from (a). The diameters of two-Ag spherical particles were fixed of $20 \mathrm{~nm}$. 
the calculated absorption and scattering efficiencies of the single Ag nanoparticle with varying particle radius were presented, shown in Figs. 1a and 1b, respectively. It was observed that with increasing $\mathrm{Ag}$ diameter from 10 to $40 \mathrm{~nm}$, absorption and scattering resonance peaks were found at the position of around $624 \mathrm{~nm}$, but the peak intensity exhibits more as the diameter increase. In Fig. 1c the optical property of Ag NPs was compared with the spectra obtained from $\mathrm{Au}$ and $\mathrm{Cu}$ nanoparticle. Clearly that the intensity of efficiency obtained from Ag nanoparticle is higher than $\mathrm{Ag}$ and $\mathrm{Cu}$ nanoparticle. For $\mathrm{Au}$ and $\mathrm{Cu}$ nanoparticle, the absorption resonance peaks are found at wavelength of $\sim 690$, and $\sim 650 \mathrm{~nm}$,
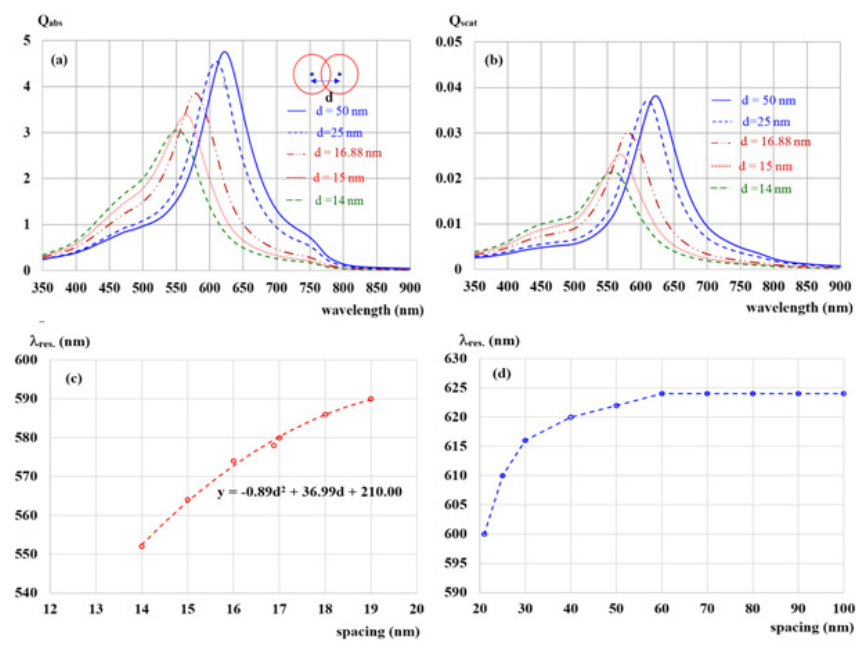

Fig. 3: Simulated of the spectral: (a) absorption and (b) scattering efficiencies as function of wavelength for two closely spaced Ag spherical particles, aligned perpendicular to both propagation direction and the linear polarized light. The spacing between two particles are indicated in the inset. (c) Shows effect of the overlapped and (d) non- overlapped distance between two Ag nanoparticles and the absorption peak position from (a).The diameters of two-Ag spherical particles were fixed of $20 \mathrm{~nm}$.

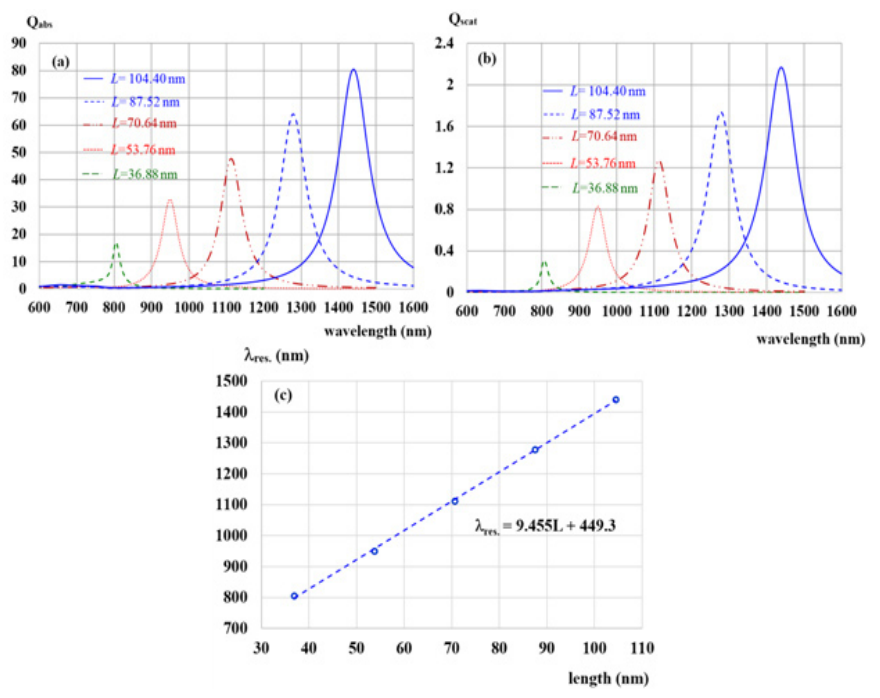

Fig. 4: Simulated (a) absorption and (b) scattering spectra of fixed $20 \mathrm{~nm}$ width Ag Nanowires aligned perpendicular to the propagation direction and parallel to the linear polarized light for the different length nanowirs, $L$. (c) Absorption peak position from (a) as a function of nanowire length(red circles)plotted with a fitted linear curve (blue curves). 
respectively, as seen in Fig. 1c. The presented results are indicated that the LSPRs are observed for Ag, $\mathrm{Au}$ and $\mathrm{Cu}$ nanoparticles in the visible wavelength region. This enhancement of in both absorption and scattering are due to the large electromagnetic field enhancement near the surface of these metal nanoparticles. In addition, the increase in the density of electrons as the size of metal nanoparticles increase results in the optical enhancement. The density of electrons in the metal nanoparticles influences the LSPRs of metal nanoparticles, which provides the enhancement of the absorption and scattering processes ${ }^{25}$.Figures 2 and 3 are the optical absorption and scattering efficiencies for the two basic two-particle geometries. In these calculation, the diameters of two spherical homogeneous particle were fixed at $20 \mathrm{~nm}$. In addition, the inter-particles distances were varied ranging from 14 to $100 \mathrm{~nm}$. Fig. 2 presents the absorption efficiencies (Fig. 2a) and scattering efficiencies (Fig. 2b) for two particles aligned perpendicular to the propagation direction and parallel to the linear polarized light. The results show as the dipolesspacing increases more, the optical efficiencies peak position shift to longer wavelength. For example it shifts approximately $\sim 1,118 \mathrm{~nm}$ for dipoles with a $14 \mathrm{nmspacing}$. By fitting the relation between optical efficiencies peak position and the dipole spacing, it found that the absorption peak shifts to longer wavelength nonlinearly with the increasing of the dipole spacing
(14-19 nm which overlaps each other) shown in Fig. 2c. The numerical calculation result was fitted by the polynomial function $\lambda_{\text {res }}[\mathrm{nm}]=-5.79 d^{\beta}+307.56 d^{R}$ $5449.50 d+33030$, where $\lambda_{\text {res }}$ and $d$ being the peak position and dipole spacing, respectively. In contrast, non-overlapping of two Ag nanospheres was found that the absorption peak shifts to longer wavelength nonlinearly with exponential-like decrease function as seen in Fig. 2d.However, as the inter-particles spacing were varied from 60 to $100 \mathrm{~nm}$, non-shifting of the absorption peak was found. Whereas, Fig. 3 are the theoretical results for two particles aligned perpendicular to both propagation direction and the linear polarized light. In contrast with another geometry, shorter wavelength-shifting was observed in these calculations down to $\sim 552 \mathrm{~nm}$ at a $14 \mathrm{~nm}$ spacing. Fig. $3 c$ shows that the absorption peak shifts to shorter wavelength nonlinearly with the increasing of the inter-particles spacing of Ag nanoparticles. The best fitted in the case of overlapping of two Ag nanospheres was found as the polynomial function $\lambda_{\text {res }}[\mathrm{nm}]=-0.895 d^{2}+36.99 d+210$. In contrast, nonoverlapping of two Ag nanospheres was found that the absorption peak shifts to longer wavelength nonlinearly with exponential-like decrease function as seen in Fig. 3d. However, as the inter-particles spacing were in the range of 60-100 nm, non-shifting of the absorption peak was found similar with the other case. Further more,Figs. 4 and 5 present the simulated absorption and scattering efficiencies

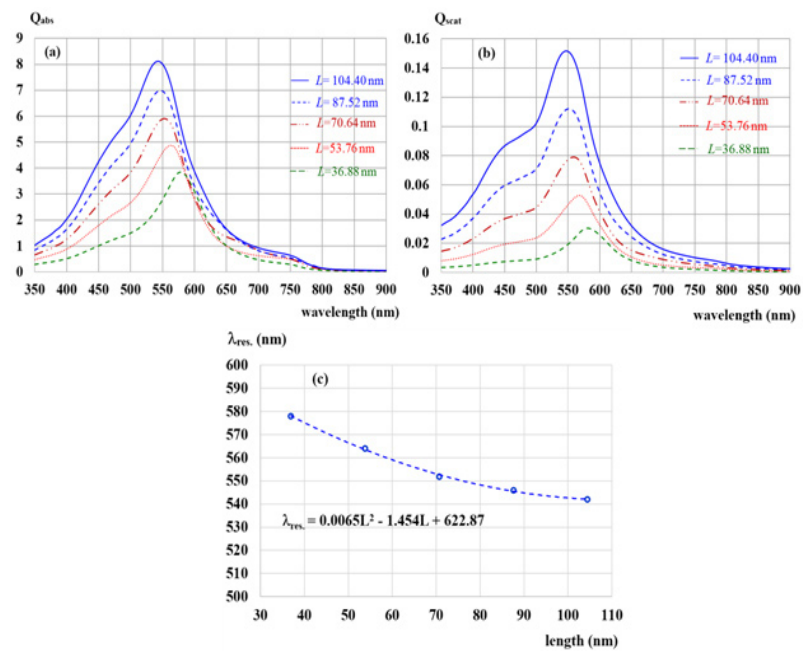

Fig. 5: Simulated (a) absorption and (b) scattering spectra of fixed $20 \mathrm{~nm}$ width Ag Nanowires aligned perpendicular to both the propagation direction and the linear polarized light for the different length nanowirs, $L$. (c) Absorption peak position from (a) as a function of nanowire length(red circles)plotted with a fitted linear curve (blue curves). 
spectra of fixed $20 \mathrm{~nm}$ width $\mathrm{Ag}$ nanowires as afunction of wavelength with different nanowire length $(L=36.88,53.76,70.64,87.52$ and 104.40 $\mathrm{nm})$. The nanowire axis in Fig. 4 was aligned perpendicular to propagation direction and parallel to the linear polarized light and bigger, whereas in Fig. 5, it was orthogonal to both propagation direction and the linear polarized light. The results was found that the longer wavelength-shifted ofthe surface plasmon both absorption and scattering peaks with increasing nanowire length for the first case as seen in Figs. $4 a$ and $4 b$, however distinct shorter wavelength-shiftedis observed for the other case shown in Figs. $5 \mathrm{a}$ and $4 \mathrm{~b}$. The maximum shifted of optical spectra in Fig. 4 was found at the wavelength of $\sim 1440 \mathrm{~nm}$ for the $104.4 \mathrm{~nm}$ nanowire length. The spectral peakposition as a function of nanowire length is shown in Fig. 4c. The best fitted was found as the linear function $\lambda_{\text {res }}[\mathrm{nm}]=9.455 L+449.3$, where $L$ is the peak position and nanowire length. Whereas, the maximum shifted of optical spectra in Fig. 5 was observed at the wavelength of $\sim 578$ $\mathrm{nm}$ for the smaller nanowire length $(L=36.88 \mathrm{~nm})$. The spectral peakposition as a function of nanowire length in this case was found as the polynomial function $\lambda_{\text {res }}[\mathrm{nm}]=0.0065 L^{2}-1.454 L+622.87$. The shifted resonance peak position can be qualitative discussed ${ }^{26}$ that due to the object in arbitrary shape in DDA model is replaced with an assembly of $N$ point dipoles. Therefore, the aligning particles axis parallel to the polarized light direction the plasma electron distributions of all particles is therefore perturbed with weakening of the repulsive forces of the field. This effect leads to a correspondingly lower resonance frequency or longer wavelength as seen in the simulated results Figs. 2 and 4. In contrast, when the excited electric field incidents normal to the longer particles axis the charge distributions of each particle acts cooperatively to enhance the repulsive action in all particles, thus decreasing the resonance wavelength as seen in Figs. 3 and 5. Moreover, the obtained results found that it is possible to shift the LSPRs frequency into the near-infrared region (NIR) of the spectrum by increase the inter-particles distance as seen in Fig. 2 and nanowire length of the silver nanoparticles as seen in Fig. 4.

\section{CONCLUSION}

In summary, the absorption and scattering efficiencies of the $\mathrm{Ag}$ nanoparticle with varying their size, shape and direction polarization of the linear polarized light were studied using the DDA method. The results found that theincorporating of $\mathrm{Ag}$ nanoparticle in the active layer of PSCsshowsstrongly LSPR induced. Moreover, the results revealed that the resonance peak of LSPR can be tuned over wavelength from the visible to the near-infrared region of the spectrum. Thus, the basic results of this study might be used for basic fabricating the more performance PSCs.

\section{ACKNOWLEDGMENTS}

The authors are grateful to the Faculty of Applied Science and Engineering, Nong Khai Campus, Khon Kaen University for all facilities supported.

\section{REFERENCES}

1. Huang, M.; Zhang, Y.; Du, C.;Peng, S.; Shi, D. Plasmonics2016, 11, 1197-1200

2. Tang, M.; Zhu, W.; Sun, L.; Yu, J.; Qian, B.; Xiao, T.Synth. Met.2015, 199, 69-73

3. Kozanoglu, D.; Apaydin, D. H.; Cirpan, A.; Esenturk, E. N. Org. Electron. 2013, 14,17201727.

4. Liu, F.; Xie, W.; Xu, Q.; Liu, Y.; Cui, K.; Feng, X.; Zhang, W.; Huang, Y. IEEE Photonics J. 2013, 5, 8400509

5. Cheng, C.-E.; Pei, Z.; Hsu, C.-C.; Chang, C.-S.; Chien, F. S.-S. Sol. Energy Mater. Sol.
Cells, 2014, 121, 80-84

6. Notarianni, M.; Vernon, K.; Chou, A.; Aljada, M.; Liu, J.; Motta, N. Sol. Energy,2014,106, 23-37

7. Barnes, W.L.; Dereux, A.; Ebbesen, T.W.Nature, 2003,424, 824-830

8. Dasri, T.; Sompech, S. Integrated Ferroelectrics, 2015, 165,176-184

9. Sompech, S.; Thaomola, S.; Dasri, T. Oriental J. Chem. 2016, 32, 85-91

10. Ranjgar, A.; Zolanvari, A.; Sadeghi, H.Plasmonics2016, 11, 277-284 
11. Dasri, T. Integrated Ferroelectrics2016, 175, 176-185

12. Viarbitskaya, S.; Cuche, A.;Teulle, A.; Sharma, J.; Girard, C.; Arbouet, A.; Erik Dujardin, E.ACS Photonics2015, 2, 744"751

13. Garjan, A. S.; Savaloni, H. EPJ B.2013, 86, 257-270

14. Cao, J.; Sun, T.; Grattan, K. T. V. Sens. Actuators, B.2014, 195, 332-351

15. Park, N. G. Mater. Today2015, 18, 65-72

16. Green, M. A.; Ho-Baillie, A.; Snaith, H. J.Nat. Photonics 2014, 8, 506-514

17. Yang, Z.; Zhang, W. H. Chin. J. Catalysis, 2014, 35, 983-988

18. Pathak, N. K.; Chander, N.; Komarala V K, Sharma, R. P.Plasmonics, 2016, 1-8

19. Cui, J.; Chen, C.; Han, J.; Cao, K.; Zhang, W.; Shen, Y.; Wang, M. Adv. Sci.2016, 3, 1500312

20. Yue, L.; Yan, B.; Attridge, M.; Wang, Z. Sol.
Energ. 2016, 124, 143-152

21. Carretero-Palacios, S.; Jiménez-Solano A.; Míguez, A.ACS Energy Letters, 2016, 1, 323331

22. Purcell, E.M.; Pennypacker, C. R. Astrophys J.1973, 186, 705-714

23. Löper, P.; Stuckelberger, M.; Niesen, B.; Werner, J.; Filipic, M.; Moon, S. -J.; Yum, J. -H.; Topic, M.; Wolf, S. D.;Ballif, C.J. Phys. Chem. Lett.2015, 6, 66-71

24. Rakiæ, A. D.; Djurišiæ, A. B.; Elazar, J. M.; Majewski, M. L.Appl. Opt.1998, 37, 52715283

25. Pradhan, A. K.; Konda, R. B.; Mustafa, H.; Mundle, R.; Bamiduro, O.; Roy, U. N.; Cui, Y.; Burger, A.Opt. Express, 2008, 16, 62026208

26. Rechberger, W.; Hohenau, A.; Leitner, A.; Krenn, J. R.; Lamprecht, B.; Aussenegg, F. R. Opt. Commun.2003, 220, 137-141 